\title{
Conditions for charge transport without recombination in low mobility organic solar cells and photodiodes (Presentation Recording)
}

\author{
Martin Stolterfoht, Ardalan Armin, The Univ. of Queensland (Australia); Bronson Philippa, \\ Ronald D. White, James Cook Univ. (Australia); Paul L. Burn, Paul Meredith, The Univ. of \\ Queensland (Australia); Gytis Juška, Vilnius Univ. (Lithuania); Almantas Pivrikas, The Univ. \\ of Queensland (Australia)
}

\begin{abstract}
Organic semiconductors typically possess low charge carrier mobilities and Langevin-type recombination dynamics, which both negatively impact the performance of organic solar cells and photodetectors. Charge transport in organic solar cells is usually characterized by the mobility-lifetime product. Using newly developed transient and steady state photocurrent measurement techniques we show that the onset of efficiency limiting photocarrier recombination is determined by the charge that can be stored on the electrodes of the device. It is shown that significant photocarrier recombination can be avoided when the total charge inside the device, defined by the trapped, dopinginduced and mobile charge carriers, is less than the electrode charge. Based upon this physics we propose the mobility-recombination coefficient product as an alternative and more convenient figure of merit to minimize the recombination losses. We validate the results in 3 different organic semiconductor-based light harvesting systems with very different charge transport properties. The findings allow the determination of the charge collection efficiency in fully operational devices. In turn, knowing the conditions under which non-geminate recombination is eliminated enables one to quantify the generation efficiency of free charge carriers. The results are relevant to a wide range of light harvesting systems, particularly those based upon disordered semiconductors, and require a rethink of the critical parameters for charge transport.
\end{abstract}

View presentation video on SPIE's Digital Library: http://dx.doi.org/10.1117/12.2186985.4519371823001 